\title{
Neutrino Splitting and Density-Dependent Dispersion Relations
}

\author{
Emilio Ciuffoli, ${ }^{1}$ Jarah Evslin, $^{1}{\text { Xiaojun } \mathrm{Bi}{ }^{2} \text { and Xinmin Zhang }}^{1,3}$ \\ ${ }^{1}$ TPCSF, IHEP, Chinese Academy of Sciences, Beijing 100049, China \\ ${ }^{2}$ Key Laboratory of Particle Astrophysics, IHEP, Chinese Academy of Sciences, Beijing 100049, China \\ ${ }^{3}$ Theoretical Physics Division, IHEP, Chinese Academy of Sciences, YuQuan Lu 19(B), \\ Beijing 100049, China \\ Correspondence should be addressed to Jarah Evslin, jarah@ihep.ac.cn
}

Received 21 August 2012; Accepted 2 October 2012

Academic Editors: G. Bonvicini, K. Cho, M. V. Garzelli, and O. A. Sampayo

Copyright (c) 2012 Emilio Ciuffoli et al. This is an open access article distributed under the Creative Commons Attribution License, which permits unrestricted use, distribution, and reproduction in any medium, provided the original work is properly cited.

\begin{abstract}
We show that particles are unstable with respect to a splitting process, which is the quantum analog of the modulational instability in anomalous dispersive media, only when their group velocity exceeds their phase velocity. In the case of a neutrino, when the concavity results from a term $E(P) \sim P^{k}$, the neutrino will decay to two neutrinos and an antineutrino after traveling a distance proportional to $E^{2+3 k}$. Unlike the Cohen-Glashow instability, the splitting instability exists even if all particles involved in the interaction have the same dispersion relations at the relevant energy scales. We show that this instability leads to strong constraints even if the energy $E$ is a function of both the momentum $P$ and also of the background density $\rho$; for example, we show that it alone would have been sufficient to eliminate any model of the MINOS/OPERA velocity anomaly which modifies the neutrino dispersion relation while leaving those of other particles intact.
\end{abstract}

\section{Motivation}

The velocity anomaly reported by OPERA [1] was the result of experimental error [2], while the weeks after OPERA's announcement were characterized by a frenzied and often mutually inconsistent attempts at profound advances in fundamental physics. However the greatest advances that were made were not the new models but the new constraints on these models. The most celebrated among these is that of Cohen and Glashow [3] who demonstrated that if at high energies the velocities of two species of particle asymptote to different values, then the fact particle will lose energy as it travels, transferring it into a succession of slow particles. Similarly it was demonstrated that the phase space for a slow particle to decay into fast particles is extremely limited [4-6]. 
These two new constraints were powerful enough to eliminate all of the models which had been formulated at the time, even those with exotic dispersion relations such as those of $[7,8]$. For example, consider the model of [7] in which the neutrino velocity is a power $P^{k}$ of the momentum $P$, with $k>0$. As we will explain, when $k>0.5$, these models were ruled experimentally by OPERA's short burst run [1] in which the energy varied by at least a factor of 5 but the velocity varied by less than a factor of 2 . On the other hand $k>2.5$ is required for consistency with supernova bounds [9-14]. However the theoretical inconsistency of this model is easily seen by extending Cohen and Glashow's calculation to the proposed dispersion relation. Such generalizations were considered in [15-18], where it was seen that they only change the decay rate by a $k$-dependent geometrical factor of order 1 . As a result the neutrino energy decays appreciably before arriving at OPERA, in contradiction with the fact that the observed spectrum agreed well with simulations up to $100 \mathrm{GeV}$ [19]. The model of [8] is consistent with the results of the short burst run, as the velocity is constant for the energy range probed. However the dispersion relation is nearly identical to that of Cohen and Glashow, with the only difference arising in a small portion of phase space at small momenta. Thus again an application of Cohen and Glashow's argument implies that in this model the neutrinos should have lost most of their energy before arriving at the OPERA detector, again in contradiction with observations.

The constraints proposed in [3-6] remain relevant even now that the velocity anomaly has disappeared, because they provide general constraints on dispersion relations that differ from those of special relativity. As is clearly explained in the first two paragraphs of [20], the dispersion relation of special relativity only applies to particles in the vacuum. In particular it applies to particles in a Lorentz-invariant vacuum. It therefore does not apply to many systems of interest, such as neutrinos traveling through the Earth or particles in the primordial plasma. If the acceleration of the universe is caused by an interacting dark energy field, then the expectation value of this field is not Lorentz-invariant, however if it is a monotonically varying scalar field then its expectation value defines a universal time. As a result, at some scale the dispersion relations of all particles will deviate from the special relativistic form. Even if dark energy is simply caused by a cosmological constant, the FRLW solution of our universe is not Lorentz-invariant, and so one may expect a combination of Hubble and Planck scale corrections to the relativistic dispersion relations.

More immediately, neutrinos traveling inside of media still exhibit a number of anomalies. For example there is a solar neutrino deficit at low energies [21, 22], and both AMANDA and IceCube observe a surplus of horizontal neutrinos in ice [23]. LSND, whose baseline is dominated by Earth and iron shielding, has seen an anomalous neutrino deficit [24] while KARMEN, whose baseline is dominated by air, has not [25]. Needless to say, these media are not Lorentz-invariant, and so the neutrino dispersion relations inside of them, even within the context of the standard model [26, 27], are not Lorentz-invariant inside of them. While it is not yet clear whether these anomalies are due to new physics, they motivate a better understanding of constraints on neutrino dispersion relations in general and in particular of neutrino dispersion relations in media.

While the constraints [3-6] are quite powerful, eventually models of superluminal neutrinos were created which avoided the assumptions implicit in these works and so avoided the constraints. The most obvious assumption is 4-momentum conservation, and discussions of escapes along those lines first appeared in [28-34]. It was also argued that one could escape by rendering other particles, such as the electron, superluminal $[35,36]$ after all the dispersion relations could well be density dependent [37-43] and all strong constraints on electron velocities appear to come from experiments in a vacuum [42, 43]. Another 
escape route is if, while traveling, the neutrinos are noninteracting, they are taking a shortcut through another dimension [44-47] or converted into a sterile flavor [48-51]. This leads one to ask if further constraints are available which may eliminate some of these other models.

\section{Neutrino Splitting}

\subsection{What Is Neutrino Splitting?}

In this note we will argue that neutrino splitting [52-54]

$$
v \longrightarrow 2 v+\bar{v}
$$

provides a powerful constraint, capable of eliminating some models which are consistent with the others. Like the Cohen-Glashow and phase space constraints, the applications of this constraint extend far beyond OPERA. The Cohen-Glashow process is only allowed if two different kinds of particles have different dispersion relations. Therefore it is avoided in models with universal high energy dispersion relations such as [35, 36, 42, 43]. While qualitative features of this model were discussed in $[35,36]$ which provide concrete models, considering interface conditions between regions with different densities and demonstrating fifth force constraints can be satisfied in those regions. The phase space constraint $[42,43]$ uses the kinematics of the creation of the neutrinos to limit its propagation along the baseline. However these neutrinos are created in a vacuum whereas the baseline is almost entirely solid rock; therefore even a small amount of environmental dependence is sufficient to eliminate these constraints. In other words, the constraint of [4-6] is satisfied in any theory in which the neutrinos are luminal in a vacuum, such as those of $[35,36]$.

The neutrino splitting constraint is more robust. It depends only on the dispersion relations of the neutrinos themselves, so it cannot be avoided by modifying the properties of the other particles. Furthermore the splitting process can occur anywhere along the baseline, so unlike the phase space constraint of [4-6] which only constrains the dispersion relations at the point where the neutrinos are created, the splitting constraint constrains the dispersion relations everywhere that the neutrinos travel and so in the region in which the velocity anomaly is claimed.

When can splitting occur? Consider a field $\phi$. Whenever the low energy effective theory has an effective vertex of the form $\phi^{k}$ with $k>2$, there is a process in which a quantum of the field $\phi$ can decay into $k-1$ quanta of $\phi$. If the field carries a conserved charge, then charge conservation does not allow an effective vertex of the form $\phi^{k}$ (if the conserved charge generates a cyclic group of finite order $N$, then this remains true so long as $N$ does not divide $k)$. However, a vertex which includes $k$ copies of the field and $k$ of the antifield is allowed. For example in the case of neutrinos the 4 neutrino Fermi vertex is allowed, which yields the desired process (2.1).

\subsection{Splitting Kinematics}

When is splitting kinematically allowed? Consider a particle with a dispersion relation $E\left(P_{x}\right)$ where $E$ is the energy and $P_{X}$ is the momentum in the $x$-direction. The energy may depend on other quantities, such as the momenta in the other directions, the background density, and the classical expectation values of various background fields. At each value of $P_{x}$ we will 
fix each of these quantities. This is not a limitation because the different particles involved in the splitting will have different values of $P_{x}$, and so the other quantities may be chosen independently for each particle involved; the constraint is only technical as it allows the dispersion relation to be expressed as a function of a single variable. Note that while the following will apply to any choice of the other variables, the reader may wish to focus his attention upon the special case $P_{y}=P_{z}=0$. Now we will show the following.

Claim 1. Particle splitting is only kinematically allowed when the group velocity exceeds the phase velocity, in other words if for particles with $x$-momentum $P$ such that, at some momentum $p \leq P$,

$$
\frac{\partial E}{\partial p}>\frac{E}{p}
$$
linear:

Intuitively this can be argued as follows. Imagine that the dispersion relation $E(p)$ is

$$
E(p)=\frac{E}{P} p
$$

Then a particle of initial energy $E$ and $x$-momentum $p$ can decay into particles of energies $x_{i} E$ and momenta $x_{i} p$ where $\sum x_{i}=1$. These decay products satisfy the linear dispersion relation because $E\left(x_{i} p\right)=x_{i} E(p)$. Although it is only marginally unstable, there is no phase space available for this decay, because any $y$-momentum for a decay product will lead to a higher energy which cannot be compensated by any change in the other particles, so no $y$-momentum for any particle is allowed, and so the phase space of kinematically allowed decays has measure zero. Therefore to make the splitting kinematically allowed one needs to reduce the energy for some of the decay products; in other words one must reduce the function $E(p)$ at some $p<P$ below the line (2.3), so that energy is available to create $y$ momenta and so phase space is available for the decay. However as the momentum increases, at or before $p=P$, the energy $E(p)$ must catch back up to the line $(2.3)$ as $E(P)=E$ is on the line. When it catches back up, up to an arbitrarily small correction, the slope of the curve (the group velocity) will be greater than that of the line (the phase velocity).

This result may be shown rigorously as follows. Consider a process in which a particle with an initial energy $E$ and $x$-momentum $P$ splits into $k$ on-shell particles with energies $E_{i}$ and $x$-momenta $P_{i}$. The dispersion relation yields $E\left(P_{i}\right)=E_{i}$. Conservation of momentum and energy yield

$$
\sum_{i=1}^{k} E_{i}=E, \quad \sum_{i=1}^{k} P_{i}=P .
$$

Combining these relations one finds

$$
0=E-E=\sum_{i=1}^{k}\left(E\left(P_{i}\right)-P_{i} \frac{E}{P}\right)
$$


which means that either for all $i, E\left(P_{i}\right)=P_{i}(E / P)$ in which case the dispersion relation is linear (2.3) and so the particle will never decay because it will be only marginally unstable or else it implies that for some values of $i$ the quantity

$$
Q\left(P_{i}\right)=E\left(P_{i}\right)-P_{i} \frac{E}{P}
$$

is positive and for others it is negative.

Now $P>P_{i}$ for all $i$ and $Q(P)=E-E=0$. Therefore the function $Q(p)$ of the momentum $p$ oscillates between positive and negative values when $p<P$ ending at $Q(P)=0$. Thus there will be some momentum $p_{0}$ when the energy reaches the line (2.3) from below, corresponding to a zero of $Q$ at which $Q$ has a positive derivative. At $p_{0}-\epsilon$ for $\epsilon$ sufficiently small the derivative of $E$ (the group velocity) is greater than that of the line (the phase velocity) completing the demonstration.

What about the converse, does a group velocity faster than the phase velocity imply that the splitting process is kinematically allowed? If the group velocity is faster than the phase velocity at some momentum $P$, then at momenta $p$ below $P$, the energy $E(p)$ will lie below the curve (2.3). Let $P_{0}$ be the lowest of such momenta, so that for $P_{0}<p<P$

$$
E(p)<\frac{p E}{P}
$$

Then splitting is kinematically allowed so long as all of the decay products have $p>P_{0}$. The problem is that if $P_{0}>P / 3$, then conservation of momentum does not allow all neutrinos to have momenta $p>P_{0}$, since the total momentum must be $P$.

Therefore we learn that if (2.7) holds for all $p$ such that $P / 3<p<P$, then the splitting is kinematically allowed. However even if it does not, then splitting reactions of the kind

$$
a v \longrightarrow(a+b) v+b \bar{v}
$$

are allowed where $(a+2 b) P_{0}<a P$. For any choice of $b, P_{0}$, and $P$, a sufficiently large value of $a$ always satisfies this constraint. This means that a beam of neutrinos can always split if the group velocity exceeds the phase velocity, but the splitting rate may be highly suppressed if the phase velocity is only exceeded for a small portion of the set of momenta beneath $P$.

\subsection{Splitting Rate}

Below we will calculate the splitting rate precisely in a particular model. In this subsection we will provide a rough estimate of the splitting rate for neutrinos. The rate is quite similar to that of the decay rate calculated by Cohen and Glashow [3]; as the relevant process is virtually identical, one need only replace the electron-positron pair with a neutrino-antineutrino pair. There are still two vertices, leading to a factor of $G_{F}$ in the amplitude and so $G_{F}^{2}$ in the decay rate.

As we consider more general dispersion relations, there is not necessarily any limiting velocity, and so the phase space computation is in general more complicated. There are still 3 final particles, which have 12 -momentum components. The conservation of 4 momenta 
yields 4 constraints, and the mass shell conditions on the 3 particles yield 3 more, leaving $12-4-3=5$ undetermined quantities. Thus the phase space for the decay is 5-dimensional. Two of these five variables describe the fractional distribution of momenta between the 3 final particles and 3 describe the orthogonal momenta. The orthogonal momenta, as we have described, would be constrained to vanish if the dispersion relation were linear. Thus these 3 variables live inside of a region whose radius is proportional to the extra phase space provided by the nonlinearity of the dispersion relations.

The size of this extra phase space depends upon the choice of neutrino dispersion relation. For example, suppose one is interested in splitting at high energies and if at these high energies the dispersion relation is effectively $E=P+a P^{k}$ with $k>1$, where $a$ has energy dimension $[1-k]$. Furthermore consider energies low enough so that $a P^{k} \ll P$, so the relativistic term is still dominant. Now the extra energy available is proportional to $a E^{k}$, and so the phase space contribution to the decay rate is proportional to $a^{3}$.

Putting these results together, the decay rate is proportional to $G_{F}^{2} a^{3}$ which has energy dimension $[-1-3 k]$. The decay rate should have units of energy; therefore by dimensional analysis the decay rate is proportional to

$$
\Gamma \sim G_{F}^{2} a^{3} E^{2+3 k} .
$$

\section{Splitting and OPERA}

In the remainder of this note we will illustrate the power of constraints from neutrino splitting by using it to demonstrate that the neutrino velocity anomaly could not have been caused by any modified dispersion relation for the neutrino alone; in other words a model consistent with OPERAs claim would have to have also modified the dispersion relations of other particles, which would be very difficult to achieve consistently with experimental constraints.

Following $[35,39-41]$ we will consider dispersion relations $E(P, \rho)$ which are general functions of the norm $P$ of the 3-momentum and background density $\rho$. An arbitrary dependence on the density $\rho$ is included in order to avoid friction with bounds on neutrino superluminalities from SN1987A [9-14], which have plagued previous attempts at the determination of such dispersion relations [15-18]. The SN1987A bound may be satisfied by choosing the $\rho$-dependence such that at small $\rho$ neutrinos are subluminal. Alternately the density-independent case, which includes, for example, the models of $[7,8]$, is included as the special case in which the $\rho$ dependence is trivial. In what follows we will make no assumptions concerning the $\rho$-dependence; our arguments will be completely general. However we will assert that the dispersion relations of all particles except for neutrinos are as in the standard model.

To reduce the number of dispersion relations that we must consider, we use two tricks. First of all, we restrict our attention to experiments inside of the Earth's crust. Here the density is more or less constant. In a region of constant density $\rho_{0}$, the density dependence of the dispersion relation is irrelevant, and so for these experiments one need only consider a function in one variable $E(P)=E\left(P, \rho_{0}\right)$. Second, we impose a kind of monotonicity condition on $E(P)$. In practice we impose that it has at most one inflection point by expanding to second order in $P$ within a given interval; however this assumption can be weakened to allow as many as 10 inflection points without changing our conclusions. If, on the contrary, there were numerous small windows in $P$ at which the neutrino is extremely subluminal, but these 
windows were so small that no neutrino yet observed by OPERA lies inside of one, then our conclusions would be evaded.

With these restrictions on the function $E(P)$ we apply three experimental constraints. The first constraint arises from the energies of the neutrinos observed in the 2008 and 2009 OPERA runs and reported in [19]. This spectrum has two features which will be relevant here. First of all, it has a very long high energy tail, with 4 percent of events above $100 \mathrm{GeV}$. Second of all, it fits expectations from simulations remarkably, even at energies of up to $100 \mathrm{GeV}$. The high energies are relevant because the effects which will be considered are much stronger at high energy, where more phase space is available, and so they imply stronger bounds. This constrains the energy loss of the neutrinos during propagation, which places constraints on neutrino splitting and therefore as we will see on the excess of the group velocity over the phase velocity.

The second experimental constraint comes from OPERA's short extraction run, which was able to unambiguously determine, up to whatever systematic errors there may be, the anticipated arrival times of 20 neutrinos [1]. All of them arrived earlier than they would have had they traveled at the speed of light. Their velocities showed fractional excesses $\epsilon=(v-$ $c) / c$ of between 1.7 and $3.9 \times 10^{-5}$. The energies of these neutrinos are not known; indeed 14 interacted outside of the detector. Nonetheless, they can be determined statistically using the energy distribution reported in [19]. The velocities determine the derivative of the dispersion relation in the momentum regime probed by OPERA.

The final constraint that we will use is from the ICARUS experiment, whose detector is subjected to the same neutrinos as its cavemate OPERA. It did not observe any high energy, nearly collinear, $e^{-} e^{+}$pairs [55]. The resulting bound can be strengthened and extended to higher energies by results from the NOMAD experiment [56] and ICECUBE [23], but there is not yet evidence for neutrino superluminality at these energy scales, and so this will not strengthen our claim. Generalizing Cohen and Glashow's results [3] for this process to a more general dispersion relation, this places a very strong bound on $E(P)-P$.

These three constraints on the neutrino dispersion relation at OPERA energies force it, in this energy range, to, respectively, not be too concave $E^{\prime}(P) \lesssim E(P) / P$ (neutrino splitting), to have a slope of $1+\epsilon$ (neutrino superluminality), and to approximately lie in the triangle $E(P) \lesssim P$ (Cohen-Glashow Bremsstrahlung). (We do not have these bounds at lower energies, but we do not need them. We consider only neutrino splitting in which all of the resulting neutrinos are within this energy range. The inclusion of decays with products below this energy range could only increase the decay rate and so strengthen our result.) The purpose of this note is to make these bounds quantitative and so to argue that no such function exists even if the bounds are chosen very conservatively. Therefore a model which explains OPERA's superluminality claim must do more than just modify the dispersion relation of the neutrino. In the conclusion we will briefly mention some such additional features that have been considered in the literature which may render such a model consistent.

In Section 4 we will discuss the three experimental constraints that we will need to derive this argument. To derive the most robust bound, we will only assume neutrino superluminality in the energy regime in which it may be inferred from OPERA data. In Section 5 we will determine this regime and so use the bounds to determine the features of a dispersion relation which are necessary to satisfy the three constraints. We will then consider the dispersion relation which minimizes the neutrino splitting probability and show in Section 6 that nonetheless it yields so much splitting as to be incompatible with OPERA's results. 


\section{The Three Constraints}

There are many functions of the form $E(P, \rho)$. We will greatly simplify the analysis by using only constraints at a fixed value of $\rho$. More precisely, we will consider only experiments in the Earth's crust, approximating its density to be constant. In particular, as most of the travel time of neutrinos from SN1987A was in much lower density media, we will not use these to fix any constraint. Fixing $\rho$, we arrive at a function only of momentum $E_{\rho}(P)$. As we will be interested in experiments in which the momenta are roughly parallel to the surface of the Earth, we will ignore the potential directional dependence and only consider the dependence on the magnitude of the momentum. This leaves us with a function $E(P)$ of a single variable. In this section we will describe the three experimental constraints in which $E(P)$ must satisfy if no new physics is introduced apart from the modification of the neutrino dispersion relation.

\subsection{Neutrino Superluminality}

It is most likely that neutrinos are never superluminal. However, in this paper we are attempting to understand general features of models which explain OPERA's neutrino superluminality claim. Therefore we will impose that neutrinos are superluminal in the region suggested by OPERA. This is certainly not a well-defined mission. It may well be that the neutrino velocity is a function of energy which is reasonably constant but has very deep subluminal troughs which are so thin that no OPERA neutrinos have hit them, and so they have not been observed. The existence of such troughs is very difficult to falsify, as they can be made as thin as one likes and can in fact lead to a consistent dispersion relation, although one would need about 10 troughs in order to do this. Therefore we will assume that no such deep, narrow troughs in the neutrino velocity as a function of energy exist. For simplicity we will choose a constant velocity as a function of energy in the regime probed by OPERA's short extraction run. It will be clear that our result holds even if this constant is the minimum value allowed by the data, or even two or three standard deviations less. However we stress that our result is not qualitatively changed by any energy dependence of the dispersion relation, so long as the velocity function does not include at least 10 deep and narrow troughs.

The group velocity of a neutrino is given by the usual formula from classical wave mechanics:

$$
v_{g}=\frac{\partial E(P)}{\partial P}
$$

and so this superluminal velocity corresponds to the slope of $E(P)$, which is slightly greater than unity, throughout the regime probed by this second run.

\subsection{Cohen-Glashow Bremsstrahlung and ICARUS}

As explained above, if a neutrino requires more energy to arrive at a given momentum than two electrons, then phase space will be available for the neutrino to emit $e^{-} e^{+}$pairs. Each such emission reduces the neutrino energy by a factor of order 1 [3], and so if most neutrinos in any energy range experience such an emission, the spectrum will be distorted significantly. The strongest bound however comes from the high energy $e^{-} e^{+}$pairs themselves. The linear dispersion relation considered by Cohen and Glashow would have resulted in millions of 
such pairs being detected by ICARUS, but in fact none were seen [55]. This fact was used by ICARUS to derive a bound on the neutrino superluminality. They assumed the linear dispersion relation of Cohen and Glashow; although this derivation may be applied individually to neutrinos in any given momentum range, it does not depend upon the relation to the function $E(P)$ at other values of $P$. (Strictly speaking, this is only true for decays in which all momenta is transferred into the electrons, in which case beta decay constraints on the neutrino dispersion relation yield a sufficiently low energy to justify our approximation. This can be extended to a sufficient fraction of phase space so long as superluminality stays below the 1 percent level. More generally, the phase space for this decay is dominated by values of $P^{\prime}$ in the OPERA energy range. In this regime the slope of $E$ is greater than unity which means that the Cohen-Glashow process is kinematically allowed and likely sufficient alone to exclude these models.) Therefore, the conservative bound that they give

$$
\epsilon=\frac{v-c}{c}<10^{-8}
$$

for neutrinos near $20 \mathrm{GeV}$ may straightforwardly be applied to the regime of neutrinos between $40-60 \mathrm{GeV}$. In fact, the real bound in this regime is stronger, due to the higher energies which far outweigh the fact that one is only using about 10 percent of the total neutrinos in deriving the bound. But this will be more than sufficient.

Summarizing, we arrive at our second constraint. For neutrino momenta in the range of $40-60 \mathrm{MeV}, E<\left(1+10^{-8}\right) P$.

\subsection{Neutrino Splitting}

The constraints from superluminality and from ICARUS may well be reconciled by a model in which neutrinos are subluminal at low energies and superluminal at high energies [16]. In such models, $E(P)$ stays below the diagonal line $E<P$ by at first having a slope of less than unity below OPERA energies, falling below the diagonal, and having a slope greater than unity at OPERA energies without ever climbing back to $E=P$. Before reaching $E=P$, above the energies probed by OPERA, the superluminality can be turned off with $E<P$ at least up to $400 \mathrm{GeV}$, so as to be consistent with NOMAD. The region between $1 \mathrm{GeV}$ and $10 \mathrm{GeV}$ is constrained by MINOS [57], which does not convincingly demonstrate superluminal neutrinos but does convincingly demonstrate that neutrinos are not too subluminal. However, one can always compensate for this constraint by forcing neutrinos to be ever more subluminal at low energies. The only important constraint is that $E(0)$ is smaller than $1 \mathrm{eV}$, so as to be consistent with bounds from beta decay. Thus a very concave $E(P)$ may explain OPERA's observed neutrino velocity in a way which is compatible with ICARUS' lack of observed high energy electron positron pairs.

The problem with this scenario is neutrino splitting [52], which as has been emphasized in $[53,54]$ strongly constrains the function $E(P)$. The constraints in those papers do not directly apply here, as we are allowing functions of $\rho$ which can easily accommodate constraints from SN1987A, and we are not assuming any particular form of the dispersion relations. However, we will see that the applicable constraints are sufficient for our goals.

In this process a neutrino with an initial momentum $P$ and energy $E(P)$ decays into 2 neutrinos and 1 antineutrino with a total momentum $P$ and total energy $E(P)$. This is possible 
kinematically only if the function $E(P)$ is concave or more precisely if the group velocity exceeds the phase velocity at some momenta:

$$
\frac{\partial E(P)}{\partial P}>\frac{E}{P}
$$

Once the splitting process is allowed kinematically, it will slow down the neutrinos, losing an order one fraction of their momenta at each reaction. Like the Cohen-Glashow process, this will ruin the agreement between the neutrino energy spectra and that predicted by simulations in [19]. In particular it will lead to a maximum neutrino energy, but recall that we will only impose superluminality in the energy range in which OPERA has measured neutrino velocities in its short extraction run, and so we only have access to the dispersion relations in this regime. Therefore this is only in contradiction with OPERA if the energy spectrum is cut off inside of this energy regime, whereas a cutoff in the long high energy tail cannot be predicted. Finally, each of these processes will cause some deflections to the neutrino beam, making it less likely to hit the OPERA detector and therefore reducing the flux, again leading to tension with [19]. In Section 6 we will calculate the corresponding decay rate for OPERA neutrinos for dispersion relations consistent with constraints from neutrino velocity and ICARUS, and we will find that it is dramatically inconsistent with the observed neutrino spectrum.

\section{OPERA's Measurement of the Neutrino Velocity}

OPERA's neutrinos are sent to Italy from the CNGS experiment at CERN. Most of them are the decay products of pions, which are created when $400 \mathrm{GeV}$ protons ejected from the SPS in France hit a graphite target in Switzerland. The fact that the original protons have about $400 \mathrm{GeV}$ each suggests that the neutrino energy distribution will have a tail extending to about $400 \mathrm{GeV}$. As the effects discussed in this note become more important at higher energy, for example, with Cohen and Glashow predicting an energy loss rate proportional to $E^{6}$ [3], it seems likely that future OPERA runs can lead to bounds which are much tighter than those in this note. This in particular applies to processes in which neutrinos emit $\mu \bar{\mu}$ pairs, $\pi^{0}$ s, and $\gamma^{\prime}$ s.

However during OPERA's 2009, 2010, and 2011 runs, due to the fact that it was using CNGS neutrino extractions which were 20 times longer than the effect for which it was searching, velocity measurements were statistical and indirect, and so could give rather little information about the energy dependence on the neutrino velocity. The results were most consistent with an energy-independent distribution, but within two standard deviations could be fit by almost any distribution.

The statistical error due to the long neutrino extraction time was essentially eliminated when OPERA received short pulses between October 22nd and November 6th of this year, allowing it to determine the velocities of individual neutrinos. However, given the short time dedicated to this experiment before SPS needed to begin accelerating lead ions, only 20 acceptable events were produced. These events all yielded measured fractional superluminalities between 1.7 and $3.9 \times 10^{-5}$. This was interpreted as a more than $6 \sigma$ signal for neutrino superluminality, but what does it tell us about the energy dependence of this superluminality? 
To determine this, first of all, one needs to consider the energy distribution of the neutrinos seen by OPERA. This is shown in Figure 10 of [19], compared with the expected results from a simulation. As one can see, in all energy regimes the observed neutrino spectrum is very close to that predicted; therefore effects like neutrino splitting and Cohen and Glashow's electron-positron pair production must be quite small throughout this range. In particular there is a long tail at high energies. While only $0.6 \%$ of the neutrinos from CNGS have energies about $100 \mathrm{GeV}$, the fact that higher energy neutrinos interact more strongly means that these account for $4 \%$ of all OPERA neutrinos [19].

In what energy range do the 20 observed events lie? It is impossible to know, since 14 events occurred outside of the detector. However, based on the distribution in Figure 10 of [19], one can determine statistically where they are likely to have occurred. As we are only interested in establishing a bound, we can safely use a very conservative estimate and state that they all occurred between 10 and $50 \mathrm{GeV}$. What does the distribution of time delays then tell us about the energy dependence of the fractional superluminality $\epsilon=(v-c) / c$ ? Again, an energy-independent value is favored, but a linear or reciprocal relation cannot be excluded. For simplicity we will use a constant distribution at the favored value

$$
\epsilon=2.5 \times 10^{-5}
$$

However we note that the splitting rate is proportional to $\epsilon^{3}$, and so even if we used a constant value as disfavored as $10^{-5}$, this would only decrease the splitting rate by a factor of 20 which we will see is not sufficient to establish consistency. Moreover, a function which oscillates but rests within this regime, such as a linear or quadratic momentum-dependent fit, would lead to more splitting than a constant choice at the minimum value $10^{-5}$; therefore our analysis will also apply to these more general polynomial dependences.

Now we are ready to assemble the superluminality constraint from Section 4.1 with the ICARUS bound from Section 4.2. The former states that for $10 \mathrm{GeV}<P<50 \mathrm{GeV}$ the slope is $1+2.5 \times 10^{-5}$. The second states that during this entire interval it lies below the diagonal $E=P$ or if it surpasses the diagonal, it only surpasses by $10^{-8} \mathrm{P}$. Of course this $10^{-8} \mathrm{P}$ is irrelevant; if indeed $E(50 \mathrm{GeV})=\left(1+10^{-8}\right) 50 \mathrm{GeV}$, then $E(49.98 \mathrm{GeV})=49.98 \mathrm{GeV}$ and so one may simply reduce the upper limit on $P$ from $50 \mathrm{GeV}$ to $49.98 \mathrm{GeV}$ and conclude that $E<P$ everywhere. For simplicity we will state

$$
E(P)<P \quad \text { In the range } 10 \mathrm{GeV}<P<50 \mathrm{GeV} \text {. }
$$

So what happens for $P<10 \mathrm{GeV}$ ? It does not matter. We can consider neutrino splittings in which all three products have more than $10 \mathrm{GeV}$. The true rate, including all neutrinos, can only be larger, and so this establishes a lower bound on the decay rate. The decay rate of a $50 \mathrm{GeV}$ neutrino to 3 neutrinos at energies between $10 \mathrm{GeV}$ and $30 \mathrm{GeV}$ can be determined using the dispersion relation described here. Notice that this dispersion relation depends on only two things. First, its slope is determined by superluminality which is measured directly by the OPERA experiment. Next, its height receives only an upper bound, the $E=P$ line given by the constraint from ICARUS. Therefore in principle we have not determined the dispersion relation; it may always be shifted to lower energies. However such a shift will only make the dispersion relation more concave and so increase the neutrino splitting decay rate. The most conservative calculation of the decay rate corresponds to the case $E(50 \mathrm{GeV})=50 \mathrm{GeV}$. In the next subsection we will determine the decay rate for this case 


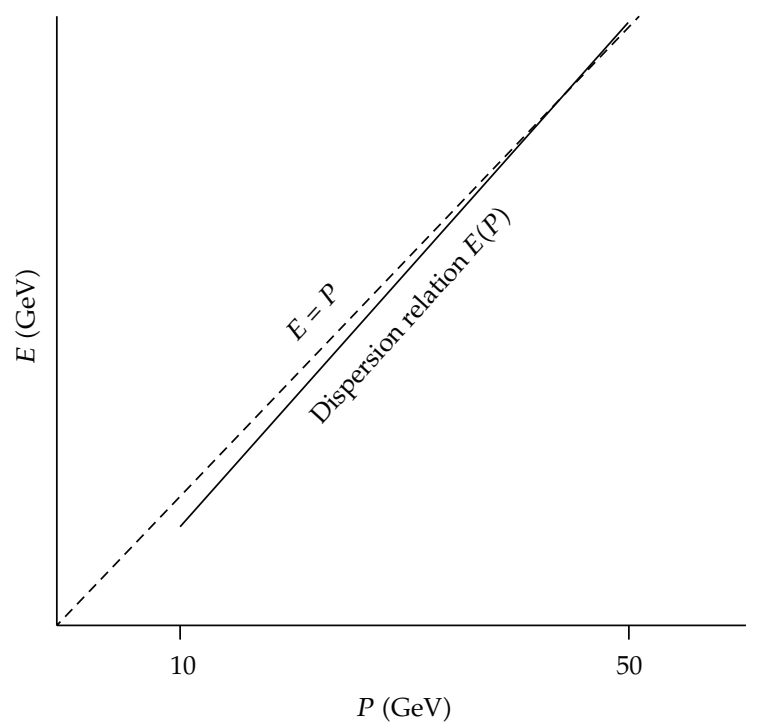

Figure 1: The dispersion relation. The velocity measurement at OPERA implies a slope of between $1+1.7 \times$ $10^{-5}$ and $1+3.9 \times 10^{-5}$ when $10 \mathrm{GeV}<P<50 \mathrm{GeV}$.

and see that indeed neutrinos almost never arrive at OPERA from CNGS without splitting, in grave contradiction with Figure 10 of [19].

\section{Splitting Decay Rate}

We assume that in the range between 10 and $50 \mathrm{GeV}$ the neutrinos can be described by the effective dispersion relation in Figure 1

$$
E(P)=(1+\epsilon)(P-B)
$$

where

$$
\epsilon \simeq 2.5 \times 10^{-5}, \quad B=\frac{\epsilon}{1+\epsilon} 50 \mathrm{GeV} \simeq 1.2 \mathrm{MeV} .
$$

This is indeed consistent with the following observations.

(1) In the range between 10 and $50 \mathrm{GeV}$, the group velocity is

$$
\left|v_{i}\right|=\left|\frac{\partial E}{\partial p_{i}}\right|=1+2.5 \times 10^{-5}
$$

(2) At $50 \mathrm{GeV}$ the neutrino 4-momentum is light-like (i.e., $E(50 \mathrm{GeV})=50 \mathrm{GeV}$ ).

Finally, we have set the neutrino masses to zero, since they are negligible at these energies. 
The Lorentz violating kinetic term that can reproduce the effective dispersion relation (6.1) is

$$
\mathcal{L}_{k}=\bar{v}\left(p_{0}+B\right) \gamma^{0} v+(1+\epsilon) \bar{v} p_{i} \gamma^{i} v
$$

where $\epsilon, B$ are the constants defined in (6.2).

\subsection{Notation}

We are interested in the splitting process

$$
v(p) \longrightarrow v\left(p^{\prime}\right)+v(k)+\bar{v}\left(k^{\prime}\right) .
$$

Let us briefly clarify our notation: we use lowercase letters for the 4-momenta $p, p^{\prime}, k$, and $k^{\prime}$ and uppercase letters for the magnitudes of the spatial vectors: $P=|\vec{p}|$. The scalar product between two 4-vectors as $p$ and $k$ will be denoted by $p \cdot k$, while if only the spatial components of the momentum are involved, we will write $\vec{p} \cdot \vec{k}$. $E_{p}$ will denote the zero component of the 4-momentum $p$ and so $p \cdot p=E_{p}^{2}-|\vec{p}|^{2}=E_{p}^{2}-P^{2}$.

\subsection{Cross-Section}

We will now calculate the decay rate for our neutral current process, neutrino splitting. The coupling between the neutrinos and the $Z$ gauge boson is

$$
\frac{g}{2 \cos \theta_{W}}=2^{1 / 4} G_{F}^{1 / 2} m_{Z}
$$

The multiplicity factor of the relevant Feynman diagram is 2, and the corresponding amplitude is given by

$$
\sum_{\text {spin }}|\mathcal{M}|^{2}=\sum_{\text {spin }} 8 G_{F}^{2}\left|\left(\bar{u}(p) \gamma_{\mu} u\left(p^{\prime}\right)\right)\left(\bar{u}(k) \gamma^{\mu} v\left(k^{\prime}\right)\right)\right|^{2}
$$

In the Lorentz-violating theory in which the kinetic term is given by (6.4), the completeness relations are

$$
\sum_{\text {spin }} u^{s}(p) \bar{u}^{s}(p)=v^{s}(p) \bar{v}^{s}(p)=\tilde{p},
$$

where

$$
\tilde{p}=\left(p_{0}+B,(1+\epsilon) p_{i}\right)
$$

Similar notation has been introduced in $[58,59]$ in this context. 
After some straightforward algebraic manipulations, we find

$$
\sum_{\text {spin }}|\mathcal{M}|^{2}=128 G_{F}^{2}\left(\tilde{p} \cdot \tilde{k^{\prime}}\right)\left(\tilde{p^{\prime}} \cdot \tilde{k}\right)
$$

and the decay width

$$
\begin{aligned}
\Gamma & =\frac{1}{2 E_{p}} \int \frac{d^{3} \overrightarrow{p^{\prime}}}{(2 \pi)^{3} 2 E_{p^{\prime}}} \int \frac{d^{3} \vec{k}}{(2 \pi)^{3} 2 E_{k}} \int \frac{d^{3} \vec{k}^{\prime}}{(2 \pi)^{3} 2 E_{k^{\prime}}} \frac{1}{2}|\mathcal{M}|^{2}(2 \pi)^{4} \delta^{4}\left(p-p^{\prime}-k-k^{\prime}\right) \\
& =\frac{8 G_{F}^{2}}{(2 \pi)^{5} E_{p}} \int \frac{d^{3} \overrightarrow{p^{\prime}}}{E_{p^{\prime}}} \int \frac{d^{3} \vec{k}}{E_{k}} \int \frac{d^{3} \vec{k}}{2 E_{k^{\prime}}}\left(\tilde{p} \cdot \tilde{k^{\prime}}\right)\left(\tilde{p^{\prime}} \cdot \tilde{k}\right) \delta^{4}\left(p-p^{\prime}-k-k^{\prime}\right)=\frac{8 G_{F}^{2}}{(2 \pi)^{5} E_{p}} \Gamma_{r} .
\end{aligned}
$$

We will restrict the domain of integration to energies between 10 and $50 \mathrm{GeV}$, since OPERA only indicates that (6.1) holds in this range. This means that the value $\Gamma$ that we will obtain is only a lower bound; however we will see that it is sufficient for our purposes. More or less following the phase space integration strategy of [15] we find

$$
\begin{aligned}
\Gamma_{r} & =\left.\int \frac{d^{3} \overrightarrow{p^{\prime}}}{E_{p^{\prime}}} \int \frac{d^{3} \vec{k}}{E_{k}} \int d^{4} k\left(\tilde{p} \cdot \tilde{k^{\prime}}\right)\left(\tilde{p^{\prime}} \cdot \tilde{k}\right) \delta\left(\tilde{k^{\prime}} \cdot \tilde{k^{\prime}}\right)\right|_{E_{k^{\prime}}>10} \delta^{4}\left(p-p^{\prime}-k-k^{\prime}\right) \\
& =\left.\int \frac{d^{3} \overrightarrow{p^{\prime}}}{E_{p^{\prime}}} \int \frac{K^{2} d K d \theta_{1} \sin \theta_{1} d \psi_{1}}{E_{k}}\left(\tilde{p} \cdot\left(\tilde{p}-\tilde{p^{\prime}}-\tilde{k}\right)\right)\left(\tilde{p^{\prime}} \cdot \tilde{k}\right) \delta\left(\left|\tilde{p}-\tilde{p^{\prime}}-\tilde{k}\right|^{2}\right)\right|_{E_{p}-E_{p^{\prime}}-E_{k}>10},
\end{aligned}
$$

where $\left|\tilde{p}-\tilde{p^{\prime}}-\tilde{k}\right|^{2}=\left(\tilde{p}-\tilde{p^{\prime}}-\tilde{k}\right) \cdot\left(\tilde{p}-\tilde{p^{\prime}}-\tilde{k}\right)$.

It is convenient now to define $\theta_{1}$ as the angle between $\vec{k}$ and $\vec{p}-\overrightarrow{p^{\prime}}$ and perform the change of variables $x=\cos \theta_{1}$. Similarly, we let $\theta_{2}$ be the angle between $\vec{p}$ and $\overrightarrow{p^{\prime}}$ and $y=$ $\cos \theta_{2}$. We will need the relations

$$
\begin{gathered}
\vec{p} \cdot \vec{k}=P K \frac{\left(P-P^{\prime} y\right) x+P^{\prime} \sqrt{1-x^{2}} \sqrt{1-y^{2}} \cos \psi_{1}}{\left|\vec{p}-\vec{p}^{\prime}\right|}, \\
\overrightarrow{p^{\prime}} \cdot \vec{k}=\vec{p} \cdot \vec{k}-\left(\vec{p}-\overrightarrow{p^{\prime}}\right) \cdot \vec{k}=\vec{p} \cdot \vec{k}-\left|\vec{p}-\overrightarrow{p^{\prime}}\right| K x .
\end{gathered}
$$

We can write the last delta function as $\delta(f(x))$ where the function $f(x)$ has a single root

$$
x_{0}=\frac{\left|p-p^{\prime}\right|^{2}-\left(P-P^{\prime}+2 B\right)^{2}+2 k\left(P-P^{\prime}+2 B\right)}{2 K\left|\vec{p}-\overrightarrow{p^{\prime}}\right|},
$$

and its first derivative reads

$$
f^{\prime}(x)=2(1+\epsilon)^{2} k\left|\vec{p}-\overrightarrow{p^{\prime}}\right| .
$$


After integrating over $x,(6.12)$ reads

$$
\Gamma_{r}=\left.\int \frac{d^{3} \overrightarrow{p^{\prime}}}{E_{p^{\prime}}} \int \frac{K d K d \psi_{1}}{E_{k}} \frac{\left(\tilde{p} \cdot\left(\tilde{p}-\tilde{p^{\prime}}-\tilde{k}\right)\right)\left(\tilde{p^{\prime}} \cdot \tilde{k}\right)}{2\left|\vec{p}-\overrightarrow{p^{\prime}}\right|}\right|_{E_{p}-E_{p^{\prime}}-E_{k}>10, x=x_{0}}
$$

The domain of integration is determined by the conditions $-1 \leq x_{0} \leq 1$. Moreover, our phase space is limited to the region in which all the neutrinos have energy between 10 and $50 \mathrm{GeV}$, yielding the additional conditions

$$
\begin{gathered}
P-P^{\prime}-K>10 \mathrm{GeV}, \\
K>10 \mathrm{GeV} .
\end{gathered}
$$

Notice that in this case we have neglected the contributions to the energy proportional to $\epsilon$. Indeed, following the same argument used in the previous section, one can easily see that they are equivalent to an irrelevant shift of the region of validity of our effective dispersion relation.

From the conditions on $x_{0}$ we obtain

$$
\begin{gathered}
\frac{P-P^{\prime}+2 B-\left|\vec{p}-\overrightarrow{p^{\prime}}\right|}{2} \leq K \leq \frac{P-P^{\prime}+2 B+\left|\vec{p}-\overrightarrow{p^{\prime}}\right|}{2}, \\
y>1-\frac{2 B\left(B+P-P^{\prime}\right)}{P^{\prime} P} .
\end{gathered}
$$

If the condition (6.20) is not satisfied, $x_{0} \leq 1$ implies

$$
K \geq \frac{P-P^{\prime}+2 B+\left|\vec{p}-\overrightarrow{p^{\prime}}\right|}{2},
$$

which is in contradiction with the condition (6.17). Since it easy to see that (6.17) and (6.18) are stronger bounds than (6.19), our domain of integration is given by

$$
\begin{gathered}
10 \mathrm{GeV} \leq K \leq P-P^{\prime}-10 \mathrm{GeV} \\
y>1-\frac{2 B\left(B+P-P^{\prime}\right)}{P^{\prime} P} .
\end{gathered}
$$

The conditions (6.22) also imply that $10 \mathrm{GeV}<P-P^{\prime}-10 \mathrm{GeV} \Rightarrow P^{\prime}<P-20 \mathrm{GeV}$, which yields the last bound. Choosing $P=50 \mathrm{GeV}$ and performing the integration we obtain the decay rate

$$
\Gamma=3.8 \times 10^{-19} \mathrm{GeV}
$$


and the decay length

$$
\ell=\frac{\hbar c}{\Gamma}=0.52 \mathrm{~km}
$$

This is much shorter than the observed $730 \mathrm{~km}$ distance that neutrinos travel from CNGS to OPERA. Therefore such models are strongly excluded, because the observed neutrino flux would not fit simulations as seen in Figure 10 of [19]. As in the case of [3] the decay rate at a fixed energy is proportional to the cube of the difference between the electron and neutrino dispersion relations; therefore a suppression of this process requires that these two energies be at least an order of magnitude closer. As the slope at most momenta $P$ is fixed directly by the OPERA velocity measurement, this requires that the neutrino energy curve in Figure 1 be modified by including narrow corrections in which the neutrino energy rapidly approaches the electron energy, corresponding to a massively subluminal neutrino in a very narrow momentum band. To reduce the difference between the two dispersion relations by an order of magnitude, one requires at least 10 such narrow bands. In a polynomial fit to the dispersion relation, this requires a polynomial of order much greater than 10.

\section{Future Directions}

\subsection{Applications for Neutrino Splitting}

In this note we have seen that the neutrino splitting process $v \rightarrow 2 v+\bar{v}$ places strong restrictions on the form of the neutrino dispersion relation $E(P)$, in particular bounding the concavity. The result is effectively that $E(P)$ will be linear or convex, or more precisely that the group velocity will never exceed the phase velocity. As this calculation depends only upon the neutrino sector, it may be used to place robust constraints on a theory, independently of the dispersion relations of the other particles.

It therefore has numerous applications. In this note we have seen that it implies that a change to the neutrino dispersion relation alone, even with an arbitrary density dependence, is not sufficient for consistency with OPERA and ICARUS results. There are many proposed methods of avoiding this problem, by changing aspects of the theory apart from the neutrino dispersion relation. For example, if the electron and neutrino dispersion relations are equal at high energies, as indeed is suggested by $\mathrm{SU}(2)$ gauge invariance, then the Cohen-Glashow process is kinematically forbidden. Therefore the condition that $E=P$ at $50 \mathrm{GeV}$ is relaxed, and the above inconsistency is lifted. However, in the $50-100 \mathrm{GeV}$ range one needs to consider processes like $v \rightarrow v+\pi^{0}, v \rightarrow v+\gamma$ and $v \rightarrow v+\mu+\bar{\mu}$, which are not eliminated by a modification of the electron dispersion relation. If OPERA continues to pursue short pulse runs, it may well be able to extend its superluminality results into this range. At that point one may be forced to render even more particles superluminal, as in the second paper of [35]. If all of these dispersion relations are linear and identical for all particles, this would be equivalent to a universal change in the effective metric, changing Einstein's equations in matter. More immediately, it is possible to calculate the constraints from these processes and to see if and when indeed other dispersion relations must be altered.

The concavity bounds may also be used to place upper bounds on superluminality at high energies, for example, at ICECUBE. Indeed, using the fact that $400 \mathrm{TeV}$ neutrinos are depleted by at most a factor of 2 with respect to simulations [23], one may reverse 
the argument of [52] to exclude the $\epsilon \sim E$ and $\epsilon \sim E^{2}$ behaviors which historically have been expected from effective field theories.

But more important is what can be said about lower energies. It is often said that upcoming data released from MINOS will confirm or deny OPERA's claim. Of course, this is unclear as MINOS neutrinos are about 10 times less energetic than OPERA neutrinos; perhaps the superluminality is simply too small to observe at MINOS. However if OPERA neutrinos are superluminal, and MINOS neutrinos are much less superluminal, then there is a positive second derivative in the dispersion relation between the two energy scales, and so OPERA energy neutrinos may split into MINOS energy neutrinos. It would be useful to calculate these decay probabilities and so to use OPERA's data to predict a lower bound on neutrino superluminality at MINOS. In this way, OPERA's claim will become falsifiable at MINOS. Of course, this assumes that no exotic physics is at play such as many flavors of sterile neutrinos, extradimensional shortcuts, and modified momentum conservation.

\subsection{Experimental Signatures}

In closing, we would like to mention that if indeed the neutrino dispersion relation does depend on density, but that this is consistent with the above constraints because, for example, charged lepton dispersion relations have a similar dependence, then there are several kinds of experimental signatures for which one may search.

Besides the neutrino, there are two particles that may travel long distances, of order a kilometer, through solid rock. These are the $\mu$ and the $\pi^{0}$. Millions of them, created by interactions with cosmic rays, have been detected in deep detectors such as MINOS. If their dispersion relations are modified with respect to those of other particles, one may look for signatures of this change in their decay rates inside of various media or even direct measurements of their travel time from the surface to underground detectors.

Although it may be that the dispersion relations of all particles have the same density dependence, then what signature is left? Recall that in 1979 FermiLab [60] was able to place strong bounds on neutrino superluminality, of order $5 \times 10^{-5}$, by racing neutrinos and muons a kilometer through rock. Significant error was introduced due to uncertainties involving the time lost by a muon traveling through rock. However this experiment was intrinsically more precise than experiments like OPERA and MINOS because there was no reliance on an external clock; one merely measured the time difference of the arrivals of the muons and neutrinos at the same location. This is why a long baseline was not necessary; one kilometer already gave a precision comparable to MINOS' $734 \mathrm{~km}$ experiment 25 years later.

One can do a very similar experiment racing neutrinos passing through rock and neutrinos beside them passing through a tunnel. If one is lucky enough to receive neutrinos that passed both through the rock and through the tunnel in the same extraction, then one can compare their arrival times and thus measure their relative velocities very accurately. Such coincidences will be much more frequent at MINOS' near detector after the energy increase of its neutrino beam scheduled to take place in two years' time.

\section{Acknowledgments}

J. Evslin is supported by the Chinese Academy of Sciences Fellowship for Young International Scientists (Grant no. 2010Y2JA01). E. Ciuffoli, X. Bi and X. Zhang are supported in part by the NSF of China. The authors thank Pengfei Yin for useful discussions. 


\section{References}

[1] T. Adam, N. Agafonova, A. Aleksandrov et al., "Measurement of the neutrino velocity with the OPERA detector in the CNGS beam," http://arxiv.org/abs/1109.4897.

[2] A. Zichichi, "Results from LVD-OPERA Combined Analysis: A Time-Shift in the OPERA Setup," http:/ /agenda.infn.it/getFile.py/access?resId=0\&materialId=slides\&confId=4896.

[3] A. G. Cohen and S. L. Glashow, "Pair creation constrains superluminal neutrino propagation," Physical Review Letters, vol. 107, Article ID 181803, 2011.

[4] L. Gonzalez-Mestres, "Astrophysical consequences of the OPERA superluminal neutrino," http:// arxiv.org/abs/1109.6630.

[5] X.-J. Bi, P.-F. Yin, Z.-H. Yu, Q. Yuan, and Constraints a, "Constraints and tests of the OPERA superluminal neutrinos," Physical Review Letters, vol. 107, no. 24, Article ID 241802, 5 pages, 2011.

[6] R. Cowsik, S. Nussinov, and U. Sarkar, "Superluminal neutrinos at OPERA confront pion decay kinematics," Physical Review Letters, vol. 107, no. 25, Article ID 251801, 4 pages, 2011.

[7] G. Cacciapaglia, A. Deandrea, and L. Panizzi, "Superluminal neutrinos in long baseline experiments and SN1987a," Journal of High Energy Physics, vol. 1111, p. 137, 2011.

[8] G. F. Giudice, S. Sibiryakov, and A. Strumia, "Interpreting OPERA results on superluminal neutrino," Nuclear Physics B, vol. 861, no. 1, pp. 1-16, 2012.

[9] K. Hirata, T. Kajita, and M. Koshiba, "Observation of a neutrino burst from the supernova SN1987A," Physical Review Letters, vol. 58, no. 14, pp. 1490-1493, 1987.

[10] R. M. Bionta, G. Blewitt, C. B. Bratton et al., "Observation of a neutrino burst in coincidence with supernova SN 1987a in the large magellanic cloud," Physical Review Letters, vol. 58, p. 1494, 1987.

[11] E. N. Alekseev, L. N. Alekseeva, I. V. Krivosheina, and V. I. Volchenko, "Detection of the neutrino signal from Sn1987a in the Lmc using the inr baksan underground scintillation telescope," Physics Letters B, vol. 205, pp. 209-214, 1988.

[12] I. Shelton, "Supernova 1987A in the Large Magellanic Cloud," IAU Circular No. 4316.

[13] R. Garrison and I. Shelton, "Supernova 1987A in the Large Magellanic Cloud," IAU Circular No. 4330.

[14] E. J. Wampler, J. W. Truran, L. B. Lucy, P. H. Hoeflich, and W. Hillebrandt, "Constraints on the interpretation of the neutrino experiments by the optical observations of SN 1987a," Astronomy and Astrophysics, vol. 182, no. 2, pp. L51-L54, 1987.

[15] M. Li, D. Liu, J. Meng, T. Wang, and L. Zhou, "Replaying neutrino bremsstrahlung with general dispersion relations," http://arxiv.org/abs/1111.3294.

[16] F. L. Villante and F. Vissani, "On the generality of the Cohen and Glashow constraints on the neutrino velocity," http://arxiv.org/abs/1110.4591.

[17] L.-A. Zhao and X. Zhang, "Fitting to data of superluminal neutrinos with phenomenological scenarios," http:/ /arxiv.org/pdf/1110.6577.pdf.

[18] Y. Huo, T. Li, Y. Liao, D. V. Nanopoulos, and Y. Qi, "Constraints on neutrino velocities revisited," Physical Review D, vol. 85, Article ID 034022, 2012.

[19] N. Agafonova, A. Aleksandrov, O. Altinok et al., "Study of neutrino interactions with the electronic detectors of the OPERA experiment," New Journal of Physics, vol. 13, Article ID 053051, 2011.

[20] A. Einstein, "on the electrodynamics of moving bodies," Annalen der Physik, vol. 17, p. 891, 1905, Annalen der Physik, vol. 14, pp. 194, 2005.

[21] P. C. de Holanda and A. Y. Smirnov, "Homestake result, sterile neutrinos and low energy solar neutrino experiments," Physical Review D, vol. 69, no. 11, Article ID 113002, 8 pages, 2004.

[22] P. C. de Holanda and A. Y. Smirnov, "Solar neutrino spectrum, sterile neutrinos, and additional radiation in the Universe," Physical Review D, vol. 83, no. 11, Article ID 113011, 13 pages, 2011.

[23] R. Abbasi et al., "Measurement of the atmospheric neutrino energy spectrum from $100 \mathrm{GeV}$ to 400 TeV with IceCube," Physical Review D, vol. 83, no. 1, Article ID 012001, 2011.

[24] A. Aguilar-Arevalo, L. B. Auerbach, R. L. Burman et al., "Evidence for neutrino oscillations from the observation of $\bar{v}_{e}$ appearance in a $\bar{v}_{\mu}$ beam," Physical Review D, vol. 64, no. 11, Article ID 112007, 22 pages, 2001.

[25] B. Armbruster, I. M. Blair, B. A. Bodmann et al., “Upper limits for neutrino oscillations $\bar{v}_{\mu} \rightarrow \bar{v}_{e}$ from muon decay at rest," Physical Review D, vol. 65, no. 11, Article ID 112001, 16 pages.

[26] L. Wolfenstein, "Neutrino oscillations in matter," Physical Review D, vol. 17, no. 9, pp. 2369-2374, 1978.

[27] S. P. Mikheev and A. Y. Smirnov, "Resonance amplication of oscillations in matter and spectroscopy of solar neutrinos," Soviet Journal of Nuclear Physics, vol. 42, p. 913, 1985, Yadernaya Fizika, vol. 42, pp. 1441, 1985.

[28] G. Amelino-Camelia, L. Freidel, J. Kowalski-Glikman, and L. Smolin, “OPERA neutrinos and relativity," Modern Physics Letters A, vol. 27, Article ID 1250063, 2012. 
[29] G. Amelino-Camelia, "On the fate of Lorentz symmetry in relative-locality," Physical Review D, vol. 85, Article ID 084034, 2012.

[30] F. R. Klinkhamer, "Superluminal neutrino, avor, and relativity," Physical Review D, vol. 85, Article ID 016011, 2012.

[31] Y. Ling, “A note on superluminal neutrinos and deformed special relativity," http://arxiv.org/abs/ 1111.3716.

[32] Y. Huo, T. Li, Y. Liao, D. V. Nanopoulos, Y. Qi, and F. Wang, "The OPERA superluminal neutrinos from deformed lorentz invariance," http:/ / arxiv.org/abs/1111.4994.

[33] G. Amelino-Camelia, "Particle-dependent deformations of Lorentz symmetry," Symmetry, vol. 4, pp. 344-378, 2012.

[34] G. Guo and X.-G. He, "Dispersion relations explaining OPERA data from deformed lorentz transformation," http:/ /arxiv.org/abs/1111.6330.

[35] J. Evslin, "Challenges confronting superluminal neutrino models," International Journal of Modern Physics Conference Series, vol. 10, no. 1, pp. 159-168, 2012, In: Proceedings of the Symposium on Cosmology and Particle Astrophysics (CosPA '11), Beijing, China, December 2011.

[36] A. Hebecker and A. Knochel, "The price of neutrino superluminality continues to rise," Physics Letters B, vol. 715, no. 1-3, pp. 116-120, 2012.

[37] D. B. Kaplan, A. E. Nelson, and N. Weiner, "Neutrino oscillations as a probe of dark energy," Physical Review Letters, vol. 93, no. 9, Article ID 091801, 4 pages, 2004.

[38] K. A. Olive and M. Pospelov, "Environmental dependence of masses and coupling constants," Physical Review D, vol. 77, no. 4, Article ID 043524, 11 pages, 2008.

[39] G. Dvali and A. Vikman, "Price for environmental neutrino-superluminality," Journal of High Energy Physics, vol. 2, p. 134, 2012.

[40] J. Alexandre, J. Ellis, and N. E. Mavromatos, “On the possibility possibility of superluminal neutrino propagation," Physics Letters B, vol. 706, pp. 456-461, 2012.

[41] A. Kehagias, "Relativistic superluminal neutrinos," http://arxiv.org/abs/1109.6312.

[42] T. Li and D. V. Nanopoulos, "Background dependent lorentz violation from string theory," http:// arxiv.org/abs/1110.0451.

[43] T. Li and D. V. Nanopoulos, "Dependent lorentz violation: natural solutions to the theoretical challenges of the OPERA experiment," The European Physical Journal C, vol. 72, Article ID 2044, 2012.

[44] G. Amelino-Camelia, G. Gubitosi, N. Loret, F. Mercati, G. Rosati, and P. Lipari, “Opera-reassessing data on the energy dependence of the speed of neutrinos," International Journal of Modern Physics D, vol. 20, no. 14, p. 2623, 2011.

[45] S. S. Gubser, "Superluminal neutrinos and extra dimensions: constraints from the null energy condition," Physics Letters B, vol. 705, p. 279, 2011.

[46] S. Hannestad and M. S. Sloth, "Apparent faster than light propagation from light sterile neutrinos," http://arxiv.org/pdf/1109.6282.pdf.

[47] A. Nicolaidis, "Neutrino shortcuts in spacetimeNeutrino shortcuts in spacetime," Modern Physics Letters A, vol. 27, Article ID 1250127, 2012.

[48] W. Winter, "Constraints on the interpretation of the superluminal motion of neutrinos at OPERA," Physical Review D, vol. 85, Article ID 017301, 2012.

[49] I. Y. Aref'eva and I. V. Volovich, “Superluminal dark neutrinos," http:/ /arxiv.org/abs/1110.0456.

[50] J. Bramante, "Sterile neutrino production production through a matter eect enhancement at long baselines," http://arxiv.org/abs/1110.4871.

[51] F. R. Klinkhamer, "Spontaneously broken Lorentz invariance from the dynamics of a heavy sterile neutrino," JETP Letters, vol. 95, pp. 497-500, 2012.

[52] D. M. Mattingly, L. Maccione, M. Galaverni, S. Liberati, and G. Sigl, "cosmogenic neutrino constraints on Planck-scale Lorentz violation," Journal of Cosmology and Astroparticle Physics, vol. 2010, article 007, 2010.

[53] J. M. Carmona and J. L. Cortes, "Constraints from neutrino decay on superluminal velocities," http://arxiv.org/abs/1110.0430.

[54] L. Maccione, S. Liberati, and D. M. Mattingly, "Violations of Lorentz invariance in the neutrino sector after OPERA," http:/ / arxiv.org/abs/1110.0783.

[55] M. Antonello, P. Aprili, B. Baibussinov et al., "Search for the analogue to Cherenkov radiation by high energy neutrinos at superluminal speeds in ICARUS," Physics Letters B, vol. 711, pp. 270-275, 2012.

[56] P. W. Cattaneo, "Testing the special relativity theory with neutrino interactions," Europhysics Letters, vol. 99, Article ID 51001, 2012.

[57] P. Adamson, P. Andreopoulos, K. E. Arms et al., "Measurement of neutrino velocity with the MINOS detectors and NuMI neutrino beam," Physical Review D, vol. 76, no. 7, Article ID 072005, 2007. 
[58] M. Mannarelli, M. Mitra, F. L. Villante, and F. Vissani, “Non-standard neutrino propagation and pion decay," Journal of High Energy Physics, vol. 1201, p. 136, 2012.

[59] F. Bezrukov and H. M. Lee, "Model dependence of the bremsstrahlung eects from the superluminal neutrino at OPERA," Physical Review D, vol. 85, Article ID 031901, 2012.

[60] G. R. Kalbeisch, N. Baggett, E. C. Fowler, and J. Alspector, “Comparison of Neutrino, anti-neutrino, and muon velocities," Physical Review Letters, vol. 43, no. 19, pp. 1361-1364, 1979. 

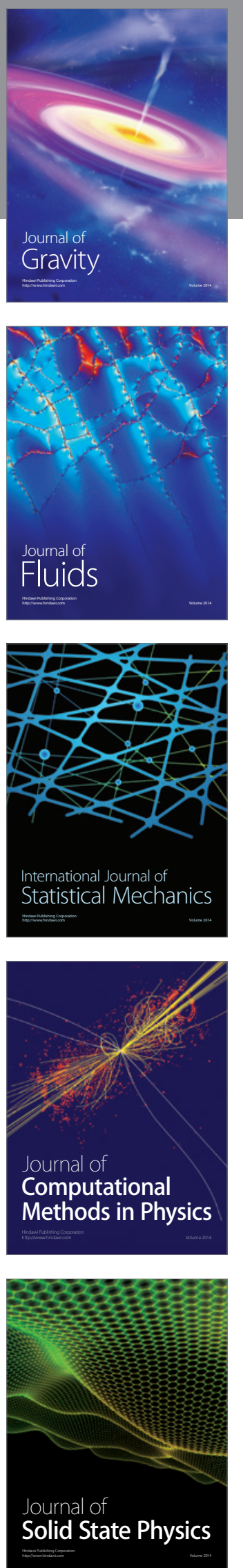
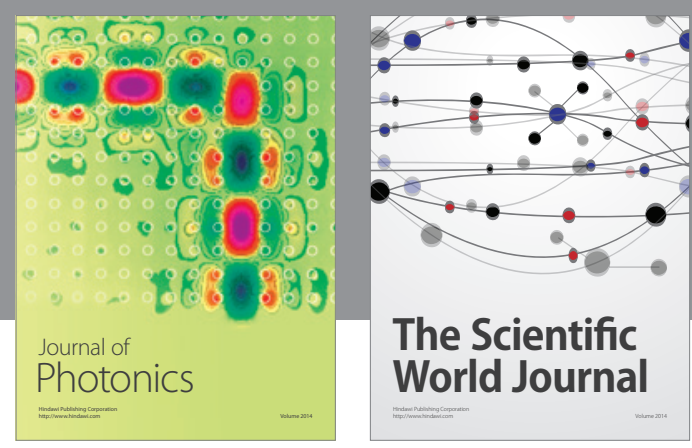

The Scientific World Journal

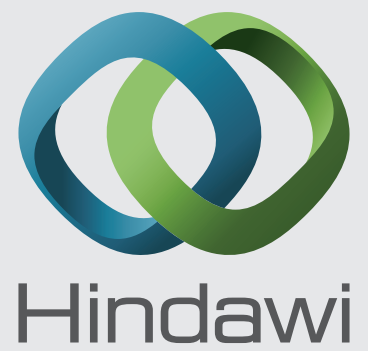

Submit your manuscripts at http://www.hindawi.com
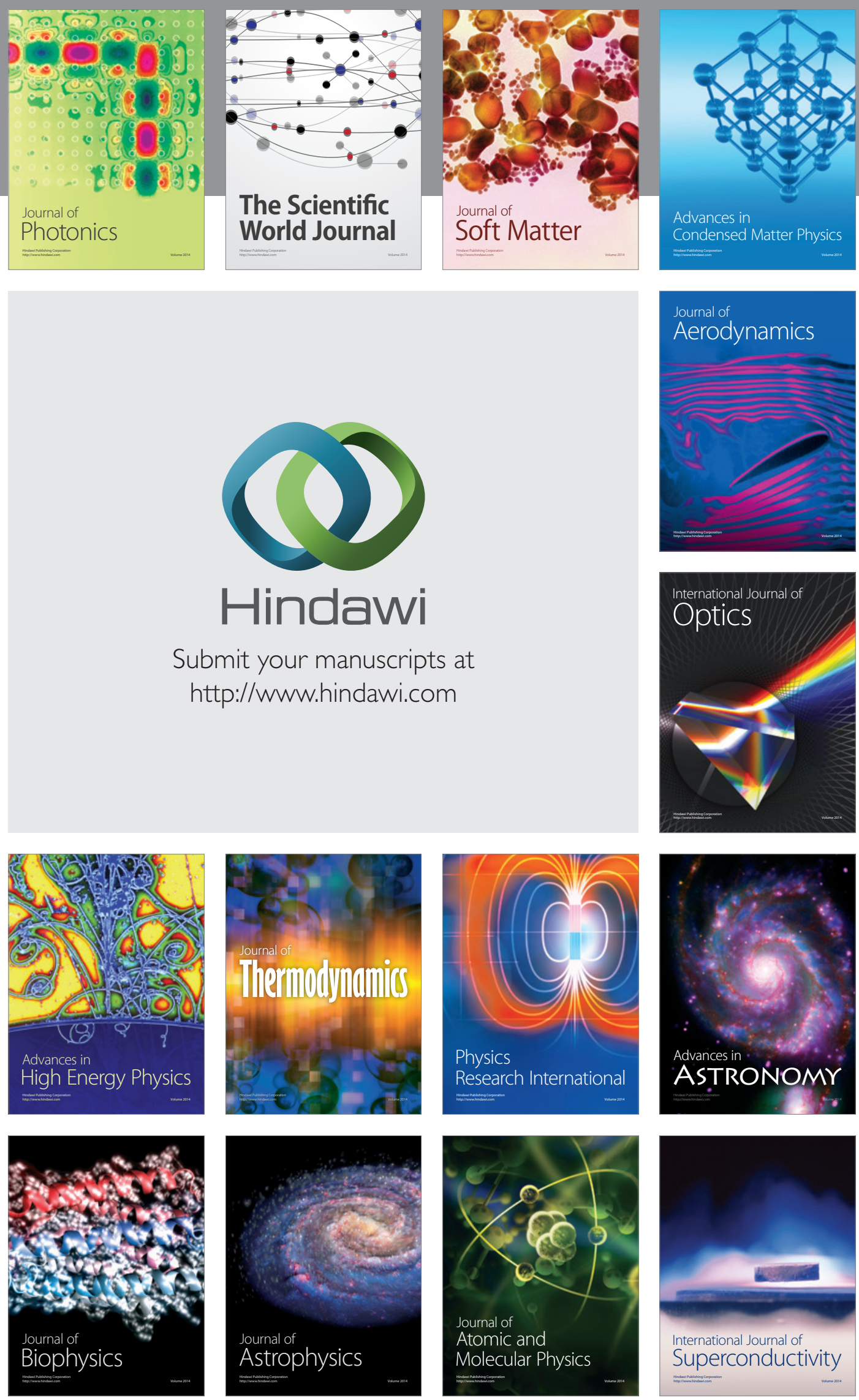
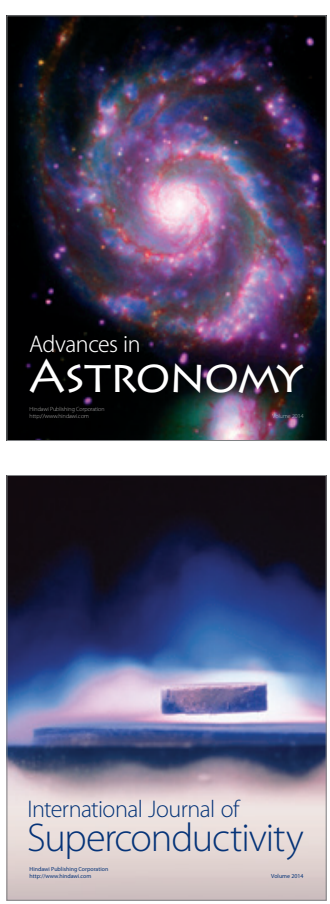